\title{
Nosocomial infection in picu: preventive strategies
}

\author{
Rabindran ${ }^{1}$, Gedam DS ${ }^{2}$ \\ ${ }^{1}$ Dr. Rabindran, Consultant Neonatologist, Billroth Hospital, Chennai; ${ }^{2}$ Dr D Sharad Gedam, Professor of bPediatrics, L \\ N Medical College, Bhopal, MP, India.
}

Address for Correspondence: Dr Rabindran, E mail: rabindranindia@yahoo.co.in

\begin{abstract}
PICU stay is largely associated with hospital acquired infection. Preventive stratigies are important to decrease morbidity and mortality associated with nosocomial infections.
\end{abstract}

Keywords: Nosocomial infection, Paediatric intensive care unit, Prevention, VAP, Bundles

Nosocomial infection is an infection acquired in hospital by a patient in whom the infection wasn't present or incubating at time of admission. It includes hospital acquired infections manifesting after discharge \& occupational infections among health workers [1]. Nosocomial infection prolongs hospital stay by 5-10 days. Common nosocomial infections in PICU are bloodstream infections (20-30\% of all infections), lower respiratory tract infections (20-35\%) \&urinary tract infections (15-20\%) [2]. Common pathogens involved are Staphylococcus aureus, coagulase negative staphylococci, E. coli,Pseudomonas aeruginosa, Klebsiella, enterococci \& candida. Bundles are checklists are available for prevention of nosocomial sepsis.

Ventilator associated Pneumonia (VAP) is a hospital acquired pneumonia developing in patients treated with mechanical ventilation for 48 hours or more who had no prior signs or symptoms of lower respiratory infection. Risk factors for VAP in children include use of opiates for sedation, sustained neuromuscular blockade,previous antibiotic therapy, technique used for endotracheal suctioning, frequent reintubation, ventilator circuit changes, gastroesophageal reflux, subglottal or tracheal stenosis, steroids \& bronchoscopy. Use of immunosuppressor drugs or neuromuscular blockers had a 4-fold \& 11-fold greater risk for VAP respectively[3].Preventive strategies of VAP include Hand hygiene, oral care, elevation ofhead end of bed, protocolised weaning, daily interruption of sedation, routine environmental decontamination with germicidal wipes, changing resuscitation bags \& circuits periodically, draining tubing condensation away from patient routinely before care \& before position changes, monitoring endotracheal tube cuff pressure (keeping it $>20 \mathrm{~cm} \mathrm{H}_{2} \mathrm{O}$ ) to avoid air leaks around the cuff which can allow entry ofbacterial pathogens into lower respiratory tract. Endotracheal tube \& gastric tube should be placed orally rather than nasally.Heated humidifiers with moisture exchangers, closed suction systems, Endotracheal tubes with extra lumen for drainage of subglottic secretions reduce the risk for VAP. Avoiding overcrowding \& understaffing, decreasing number of heel sticks \&attempts at venipuncture, using single-dose administration of medications such as albumin, avoiding drugs associated with increased risk of nosocomial infection like histamine-2 blockers \& dexamethasone, use of sterile suctioning techniques, continuous subglottic aspiration, early trace element supplementation,avoidance of gastric overdistension\& elimination of nonessential tracheal suction help to decrease VAP. A recent study shows that prophylactic probiotics decreases incidence of VAP by $77 \%$ [4].

Central line associated blood stream infection (CLABSI) is a primary blood stream infection developing in a patient with central line placed within 48 hour before onset of blood stream infection. Preventive measures include early institution of enteral feedings, discontinuation of parenteral alimentation (especially intravenous lipids) at the earliest, limitation of open catheter circuits \&heparin flushes, careful preparation of intravenous fluids, blood products \& routine change of administration sets every 72 hours (or within 24 hours if lipids are used). Upper extremity should be preferred for catheter insertion. Novel preventive techniques include use of silver sulfadiazine impregnated catheters \& cuffs, heparin-bonded 
catheters, antibiotic-coated (eg, rifampin-minocycline) catheters\&use of chlorhexidine for cutaneous antisepsis. Changing the catheter with a guidewire represented a nearly five-fold greater risk for BSI [5].

Catheter associated Urinary tract infection (CAUTI) is a major cause of nosocomial infection. Risk factors include duration of catheter placement $>14$ days, contamination of collection bag, periurethral contamination with pathogenic microorganisms \&lack of aseptic techniques during catheter placement[6]. Strategies for prevention include avoiding insertion of catheter ifpossible, early removal by checklist implementation, nurse based interventions daily, maintaining a closed drainage system with unobstructed urine flow, use of intermittent catheterization, condom catheter\& portable bladder ultrasound scanner. Prevention bundles, hand hygiene\& prudent use of antimicrobials are the major measures of prevention of nosocomial infection[7].

Choudhury et al in this issue concluded that the presence of nosocomial infection was associated with a long period of hospitalization and use of invasive devices leading to increased cost of health care. So adherence to infection control guideline laid by Center of disease control and short term use of invasive devices and judicious use of antibiotics can play important role in preventing such nosocomial infections [8].

\section{Reference}

1. SandeepTripathi.Health care quality and hospital acquired infection in Intensive care: Bundles and checklists.; BJMP 2014;7(2):a715.

2. Lodha R1, Natchu UC, Nanda M, Kabra SK.Nosocomial infections in pediatric intensive care units.Indian J Pediatr.2001 Nov;68(11):1063-70.
3. Fayon MJ, Tucci M, Lacroix J, Farrell CA, Gauthier M, Lafleur L, Nadeau D.Nosocomial pneumonia and tracheitis in a paediatric intensive care unit: a prospective study. Am J RespirCrit Care Med. 1997 Jan;155(1):162-9.

4. Banupriya B, Biswal N, Srinivasaraghavan R, Narayanan P, Mandal J.Probiotic prophylaxis to prevent ventilator associated pneumonia (VAP) in children on mechanical ventilation: an open-label randomized controlled trial. Intensive Care Med. 2015 Apr;41(4):677-85. doi: 10.1007/s00134-015-3694-4.

5. Almuneef MA, Memish ZA, Balkhy HH, Hijazi O, Cunningham G, Francis C. Rate, risk factors and outcomes of catheter related bloodstream infection in a paediatric intensive care unit in Saudi Arabia. J Hosp Infect. 2006 Feb;62(2):207-13.

6. Rosenthal V, Todi S, Álvarez-Moreno C, Pawar M, Karlekar A, Zeggwagh A, et al. Impact of a multidimensional infection control strategy on catheterassociated urinary tract infection rates in the adult intensive care units of 15 developing countries: findings of the International Nosocomial Infection Control Consortium (INICC). Infection. 2012 Oct;40(5):517-26. doi: 10.1007/s15010-012-0278-x.

7. LulRaka.Prevention and Control of Hospital-Related Infections in Low and Middle Income Countries; The Open Infectious Diseases Journal, 2010, 4, 125-131 125 1874-2793/10 2010.

8. Choudhury J, Mohanty D, Routray S S. Microbiological profile of Nosocomial infections in the pediatric patients admitted to intensive care unit: Int $\mathrm{J}$ Pediatr Res 2016;3(2):100-104.doi:10.17511/ijpr.2016.2.05.

\section{How to cite this article?}

Rabindran, Gedam DS . Nosocomial infection in picu: preventive strategies. Pediatic Review: Int J Pediatr Res 2016;3(2):80-81.doi:10.17511/ijpr.2016.102.01. 